\title{
ERRATUM
}

\section{Erratum to: Practical Approach to Initiating SGLT2 Inhibitors in Type 2 Diabetes}

Fernando Gomez-Peralta $\cdot$ Cristina Abreu $\cdot$ Albert Lecube •

Diego Bellido · Alfonso Soto · Cristóbal Morales · Miguel Brito-Sanfiel ·

Guillermo Umpierrez

Published online: August 23, 2017

(c) The Author(s) 2017. This article is an open access publication

Erratum to: Diabetes Ther

DOI 10.1007/s13300-017-0277-0

In the original publication, corresponding author e-mail address and Fig. 2 were published incorrectly. The correct e-mail address and the revised Fig. 2 are given here.

The corresponding author's e-mail address should read as fgomezp@saludcastillayleon.es.

The incorrect statement in Fig. 2 "BP $<140 /$ $80 \mathrm{mmHg}$, aged older than 65 years or hemodynamically unstable*?" should read as "BP $<140 / 80 \mathrm{mmHg}$, aged older than 65 years or hemodynamically unstable**?"

The online version of the original article can be found under doi:10.1007/s13300-017-0277-0.

F. Gomez-Peralta $(\bowtie) \cdot$ C. Abreu

Endocrinology and Nutrition Unit, Segovia General

Hospital, Segovia, Spain

e-mail: fgomezp@saludcastillayleon.es

A. Lecube

Endocrinology and Nutrition Unit, Hospital

Universitari Arnau de Vilanova de Lleida, Institut de

Recerca Biomèdica de Lleida, CIBERDEM (CIBER de

Diabetes y Enfermedades Metabólicas Asociadas,

ISCIII), University of Lleida, Lleida, Spain

D. Bellido

Endocrinology and Nutrition Section, Hospital

Marcide, Ferrol, Spain

A. Soto

Endocrinology and Nutrition Department, Hospital

de La Coruña, A Coruña, Spain
The incorrect statement in Fig. 2 "Reintroduce treatment according to clinical situation*?" should read as "Reintroduce treatment according to clinical situation ${ }^{* * * ? "}$
C. Morales

Endocrinology and Nutrition Department, Virgen

Macarena Hospital, Seville, Spain

M. Brito-Sanfiel

Endocrinology and Nutrition Department, Hospital Universitario Puerta de Hierro Majadahonda,

Madrid, Spain

G. Umpierrez

Department of Medicine, Emory University School of Medicine, Atlanta, GA, USA 
A

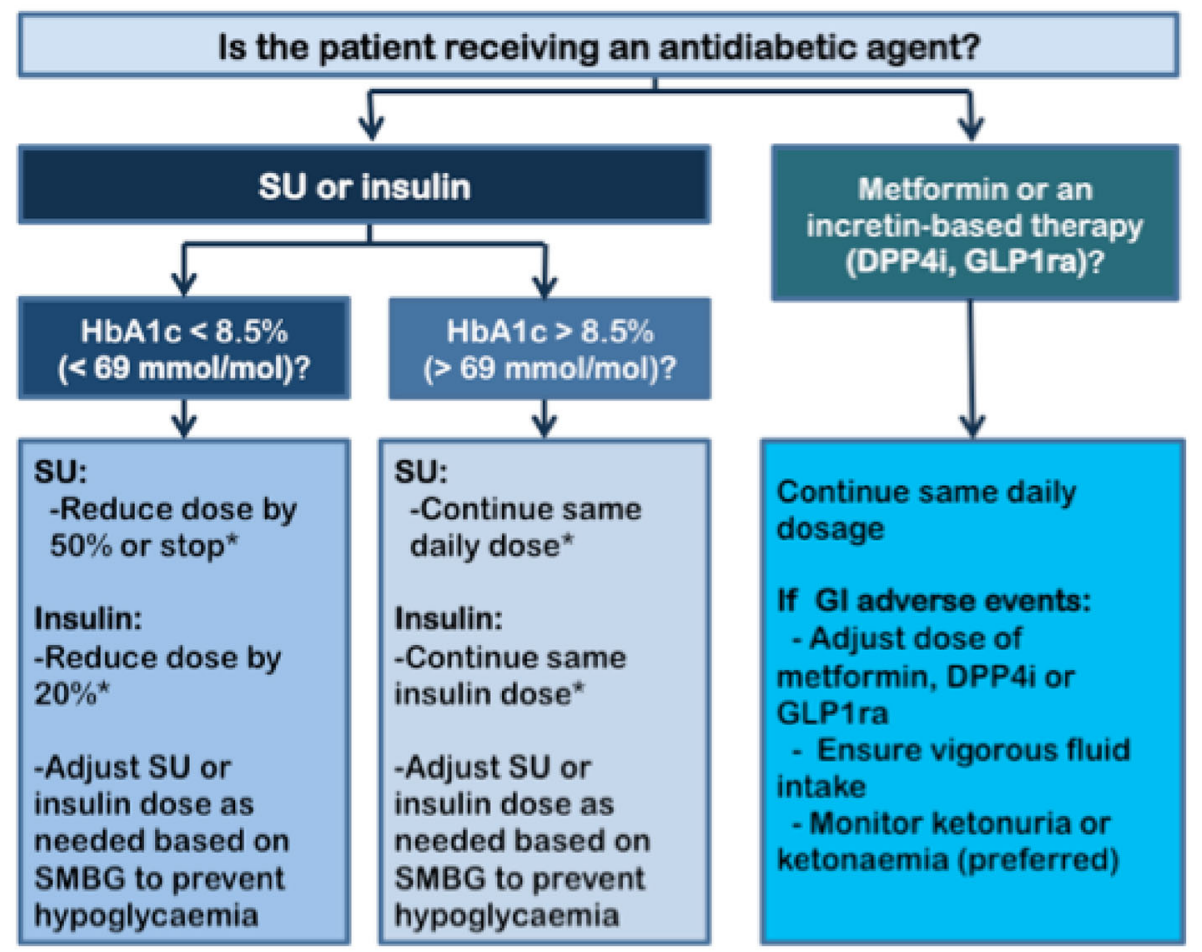

B

\begin{tabular}{|c|c|c|}
\hline \multicolumn{3}{|c|}{$\begin{array}{l}\text { Is the patient receiving a diuretic } \\
\text { or an antihypertensive treatment? }\end{array}$} \\
\hline$\sqrt{ }$ & \multicolumn{2}{|c|}{$\nabla$} \\
\hline A diuretic? & \multicolumn{2}{|c|}{ An antihypertensive? } \\
\hline \multirow[b]{2}{*}{$\downarrow$} & $\sqrt{7}$ & $\nabla$ \\
\hline & $\begin{array}{c}\text { BP }<140 / 80 \mathrm{mmHg} \text {, } \\
\text { aged older than } 65 \\
\text { years or }\end{array}$ & $\begin{array}{c}\mathrm{BP}>140 / 80 \mathrm{mmHg}, \\
\text { aged below } 65 \text { years } \\
\text { and }\end{array}$ \\
\hline \multirow{2}{*}{$\begin{array}{c}\text { - Withdrawal or } \\
\text { reduce dose by } \\
50 \%\end{array}$} & unstable"*? & stable "m? \\
\hline & $\vee$ & $\boldsymbol{\psi}$ \\
\hline $\begin{array}{l}50 \% \\
\text { - Reintroduce } \\
\text { treatment } \\
\text { according to } \\
\text { clinical situation }\end{array}$ & $\begin{array}{c}\text {-Reduce dose of } \\
\text { antihypertensive } \\
\text { treatment } \\
\text { - Revise upward } \\
\text { dosage according to } \\
\text { BP weekly } \\
\text { monitoring }\end{array}$ & $\begin{array}{l}\text {-Continue daily dose of } \\
\text { antihypertensive } \\
\text { treatment } \\
\text { - Revise the treatment } \\
\text { according to weekly } \\
\text { monitoring }\end{array}$ \\
\hline
\end{tabular}

Fig. 2 Proposed algorithm for adjusting antidiabetic agents (a) and diuretic/antihypertensive therapy (b) when initiating SGLT2 inhibitors in patients with type 2 diabetes. DPP4 $i$ DPP 4 inhibitors, GI gastrointestinal, GLPIra GLP-1 receptor agonists, $S U$ sulfonylureas, $S M B G$ self-monitoring of blood glucose, $B P$ blood pressure. ${ }^{*}$ Avoid insulin withdrawal to minimize the risk of euglycemic diabetic ketoacidosis. ${ }^{* *}$ Hemodynamically unstable defined as atrial fibrillation, orthostatic hypotension or blood pressure lability, prior syncope, etc. ${ }^{* * *}$ Clinical situation defined by congestive heart failure, edema, renal function 
Open Access. This article is distributed under the terms of the Creative Commons Attribution-NonCommercial 4.0 International License (http://creativecommons.org/licenses/ by-nc/4.0/), which permits any noncommercial use, distribution, and reproduction in any medium, provided you give appropriate credit to the original author(s) and the source, provide a link to the Creative Commons license, and indicate if changes were made. 